\title{
Comparison of Clonidine and Metoprolol Effectiveness on the Control of Post-Operative Blood Pressure in Otorhinolaryngology-Head and Neck Surgeries: A Clinical Trial
}

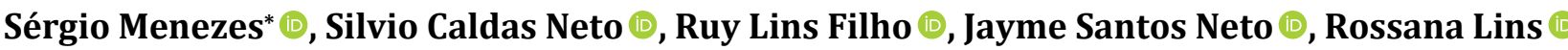 \\ Universidade Federal de Pernambuco, Recife, Brazil \\ Email:silvio_caldas@oi.com.br, rllins@uol.com.br, jaymemed@yahoo.com.br, \\ rossanalins@terra.com.br, *sergiovdsm@gmail.com
}

How to cite this paper: Menezes, S., Neto, S.C., Filho, R.L., Neto, J.S. and Lins, R. (2021) Comparison of Clonidine and Metoprolol Effectiveness on the Control of Post-Operative Blood Pressure in Otorhinolaryngology-Head and Neck Surgeries: A Clinical Trial. Open Journal of Anesthesiology, 11, 49-58.

https://doi.org/10.4236/ojanes.2021.112006

Received: January 21, 2021

Accepted: February 20, 2021

Published: February 23, 2021

Copyright $\odot 2021$ by author(s) and Scientific Research Publishing Inc. This work is licensed under the Creative Commons Attribution International License (CC BY 4.0).

http://creativecommons.org/licenses/by/4.0/

\begin{abstract}
Objectives: Adequate control of hemodynamic parameters in patients undergoing otolaryngology-head and neck (OLHN) surgeries is one of the objectives of peri-operative anesthesia management. In this context, we seek to evaluate the effectiveness of metoprolol compared to clonidine in the postoperative control of systolic (SBP) and diastolic (DBP) blood pressures, and heart rate (HR), when these medications are used in the immediate pre-operative period. Method: this is a randomized double-blind clinical trial. Patients over 18 years old, according to the American Society of Anesthesiologists Physical Status Classification (ASA) I or II, who would undergo OLHN surgery, presenting with greater than $140 \mathrm{mmHg}$ (SBP) or $90 \mathrm{mmHg}$ (DBP), upon arrival at the surgical ward, were included in the study. 46 patients were randomized into two groups ( $\mathrm{C}$ or $\mathrm{M}$ ) who received clonidine $(75$ or $150 \mathrm{mcg}$ ) or metoprolol (5 or $10 \mathrm{mg}$ ), respectively. The averages of SBP, DBP and HR were compared between groups upon arrival at the post anesthesia care unit (PACU) and four hours post-surgery. Results: In group C, SBP (127.7 \pm 18.8 vs $137.3 \pm 14.1 \mathrm{mmHg}, \mathrm{p}=0.03)$ and $\mathrm{DBP}(73.1 \pm 15.46$ vs $82.6 \pm 7.9 \mathrm{mmHg}$, $\mathrm{p}<0.01)$ were lower, when compared with group $M$. No difference was observed in HR immediately upon PACU arrival. In addition, for four hours post-surgery, no change was noted in the SBP, DBP or HR. Conclusion: metoprolol was less effective than clonidine in reducing systolic and diastolic blood pressures in the immediate post-operative period of OLHN surgeries.
\end{abstract}

\section{Keywords}

Clonidine, Metoprolol, Hypertension, Post-Operative 


\section{Introduction}

Otorhinolaryngology-head and neck surgeries encompass a wide variety of surgeries that have in common the need for strict control of blood pressure in the peri-operative period. The higher the pressure, the greater the bleeding in the operative field, increasing the incidence of complications directly related to the difficulty in visualizing anatomical structures [1] [2] [3].

Arterial hypertension is also an important risk factor for post-operative bleeding [4] and its incidence in the post anesthesia care unit (PACU) in these surgeries is around $30 \%$, varying according to pre-operative clinical conditions, type of surgery performed and medications used by the patient in the peri-operative period [5].

Post-operative bleeding implies several undesirable events. These events may range from patient apprehension as a result of constant dressing changes, to the possibility of large bruises requiring surgical re-intervention. Procedures such as tamponade, cauterization or reoperation are sometimes necessary to control bleeding, and, depending on the volume and surgical site, it can lead to patient's death. Hemorrhage after thyroid surgery could provoke airway involvement by compression of the hematoma. For this surgery, one of the main risk factors is post-operative hypertension [6].

The intra-operative control of blood pressure (BP) and heart rate (HR) with the use of clonidine or metoprolol has been demonstrated in several studies. The reduction of these hemodynamic parameters, notably in endoscopic nasal surgeries, improves the visualization of the surgical field due to less bleeding [7] [8] [9]. However, the literature is scarce regarding the effectiveness of post-operative BP and HR control of these medications in OLHN surgeries.

Clonidine has several useful characteristics, in addition to its hypotensive action, it works as a sedative and anxiolytic agent, anesthetic adjuvant, promotes an analgesic effect, prevents post-operative nausea and vomiting (PONV) and reduces tremor and agitation [10].

On the other hand, metoprolol has been chosen for its particular characteristic of providing protection against cardiovascular events in the peri-operative period, such as ischemia and myocardial infarction. In addition, some studies suggest that this drug promotes greater HR reduction, which is more important for the control of intra-operative bleeding [1] [11].

Therefore, both clonidine and metoprolol can be used for intra-operative BP and HR control in order to reduce bleeding, each presenting its own characteristics and advantages. However, there is no definition of which of these medications would be the most effective for controlling $\mathrm{BP}$ and $\mathrm{HR}$ in the post-operative period, in order to reduce the incidence of hemorrhagic complications.

Thus, the primary objective of this study was to assess whether metoprolol is as effective as clonidine in post-operative BP control upon arrival at PACU.

\section{Methods}

This study was a randomized, double-blind, phase IV clinical trial approved by 
the Ethics Committee (CAAE 79705417.4.0000.5208, report \#: 2,514,730). Clinical trial registration at http://www.ensaiosclinicos.gov.br/, trial RBR-6S3KKR. The universal trial number is U1111-1211-7890.

The study was carried out from June 2018 to December 2019, at a University Hospital (Hospital das Clínicas da Federal University of Pernambuco). All patients who would undergo OLHN surgeries were assessed for eligibility criteria upon arrival at the operating room. Patients between the ages of 18 and 65 years, with American Society of Anesthesiology (ASA) physical status of I or II, whose SBP was greater than or equal to $140 \mathrm{mmHg}$ or DBP was greater than or equal to $90 \mathrm{mmHg}$ at the time of recruitment (regardless of previous diagnosis of systemic arterial hypertension). Patients with HR below 60 beats per minute, patients with atrioventricular block, severe asthma, past allergies to clonidine or metoprolol or patients using pacemakers were excluded from the study.

The randomization of the study was performed with the help of the randomizer.org website, in which a random sequence is generated to designate which of the two treatment arms the patients would be allocated to. After signing the informed consent, a research assistant (a physician), who did not participate in the anesthetic procedure or in the patient's evaluation, opened sequentially numbered, opaque, sealed envelopes. The envelopes had an indication of whether the patients would be allocated to group C (clonidine) or group M (metoprolol). The same assistant administered the medications according to the following protocol:

In both groups, the patients were lying in the supine position, on a bed in the pre-anesthesia room, monitored by a cardioscope, pulse oximeter and non-invasive blood pressure measuring device.

In group $\mathrm{C}$, patients received intravenous clonidine at a dose of $75 \mu \mathrm{g}$. Similarly, in group $\mathrm{M}$, patients received intravenous metoprolol, at a dose of $5 \mathrm{mg}$. In both groups, all patients were reassessed after 20 minutes. Those who still had SBP greater than or equal to $140 \mathrm{mmHg}$ or DBP greater than or equal to 90 $\mathrm{mmHg}$ received an additional dose of $75 \mu \mathrm{g}$ of intravenous clonidine (group C) or $5 \mathrm{mg}$ of metoprolol (group $\mathrm{M}$ ). In both groups, before the administration of the second dose, if the HR was less than 60 beats per minute, the new dose would not be administered.

Subsequently, the patient was taken to the operating room, and after monitoring by a cardioscope, pulse oximeter and non-invasive blood pressure measuring device, balanced general anesthesia was administered by an anesthesiologist who did not know which group the patient was allocated to. This anesthesiologist was part of the research team, and was instructed on the collection of patient data, and on the standardization of anesthetic drugs.

This was followed by intravenous administration of propofol 1 to $2 \mathrm{mg} / \mathrm{kg}$, fentanyl $5 \mu \mathrm{g} / \mathrm{kg}$, rocuronium $0.6 \mathrm{mg} / \mathrm{kg}$, lidocaine $1 \mathrm{mg} / \mathrm{kg}$, and sevoflurane for maintenance. The use of remifentanil and additional doses of rocuronium were at the discretion of the assistant anesthesiologist. For post-operative analgesia, 
tramadol $100 \mathrm{mg}$, ketoprofen $100 \mathrm{mg}$ and dipyrone $2 \mathrm{~g}$ were administered, and for prophylaxis of post-operative nausea and vomiting, ondansetron $4 \mathrm{mg}$ and dexamethasone $10 \mathrm{mg}$. After the end of anesthesia, the patients were taken to the post-anesthetic recovery room (PACU) where they remained for a minimum of one hour under the care of another anesthesiologist.

We recorded the patients' pre-operative data regarding age, sex, weight, height, ASA classification and presence of comorbidities, to characterize the sample. In addition, we also recorded if there was a need to administer a second dose of clonidine or metoprolol and the initial values (arrival to the surgical room) of SBP, DBP and HR. As outcomes, we recorded SBP, DBP and HR upon arrival at the PACU (primary outcome), as well as SBP, DBP and HR 4 hours post-surgery. We also recorded the rate of patients who were normotensive upon arrival to the PACU (defined as patients with $\mathrm{SBP}<140 \mathrm{mmHg}$, or DBP $<$ $90 \mathrm{mmHg}$ ). Surgical complications that could be related to excessive bleeding were also noted, as well as the need for surgical re-intervention or records of excessive bleeding in the post-operative period. Other complications that could be associated with the use of metoprolol or clonidine, such as bronchospasm or atrioventricular heart block, were also recorded. The incidence of arterial hypotension (defined by SBP less than $90 \mathrm{mmHg}$ or DBP less than $40 \mathrm{mmHg}$ ), bradycardia less than $50 \mathrm{bpm}$, and the use of vasopressors or atropine up to 4 hours after surgery were also documented.

In order to calculate the sample size, 46 patients would be necessary to verify a minimal clinically important difference of $10 \%$ between the pressure means of the groups (standard deviation of $15 \mathrm{mmHg}$ ), with a $95 \%$ confidence interval (unicaudal t-test, as it is a non-inferiority trial), and power of $80 \%$. For the hypothesis test, blood pressures were assessed by the t-student test, while for the dichotomous categorical variables, the Chi-square exact test was used. Except when the sample count was less than 5 or the categorical variables were ordinal, then Fisher's test was used.

\section{Results}

Of the 60 patients assessed for eligibility, 46 were selected and randomized. There were three post-randomization losses ( 1 loss of follow-up in each group and 1 patient in group $\mathrm{M}$ who did not receive the intervention because their surgery was canceled). 43 patients were included as shown in Figure 1. The groups were similar in terms of demographic characteristics, ASA classification, comorbidities and surgical time, as described in Table 1.

There was also no statistical difference regarding the initial measurements of SBP, DBP and HR upon arrival at the operating room, between the groups (Table 2). In group $M$, the administration of a second dose was more frequent: ten patients, while in group $\mathrm{C}$, only two patients needed it $(\mathrm{p}<0.01)$.

As for the primary objective, in group $\mathrm{C}$, the mean SBP $(127 \pm 18 \mathrm{mmHg})$ was lower than in group $\mathrm{M}(137 \pm 14 \mathrm{mmHg})$, (difference in means: $10 \mathrm{mmHg}$; $95 \%$ 


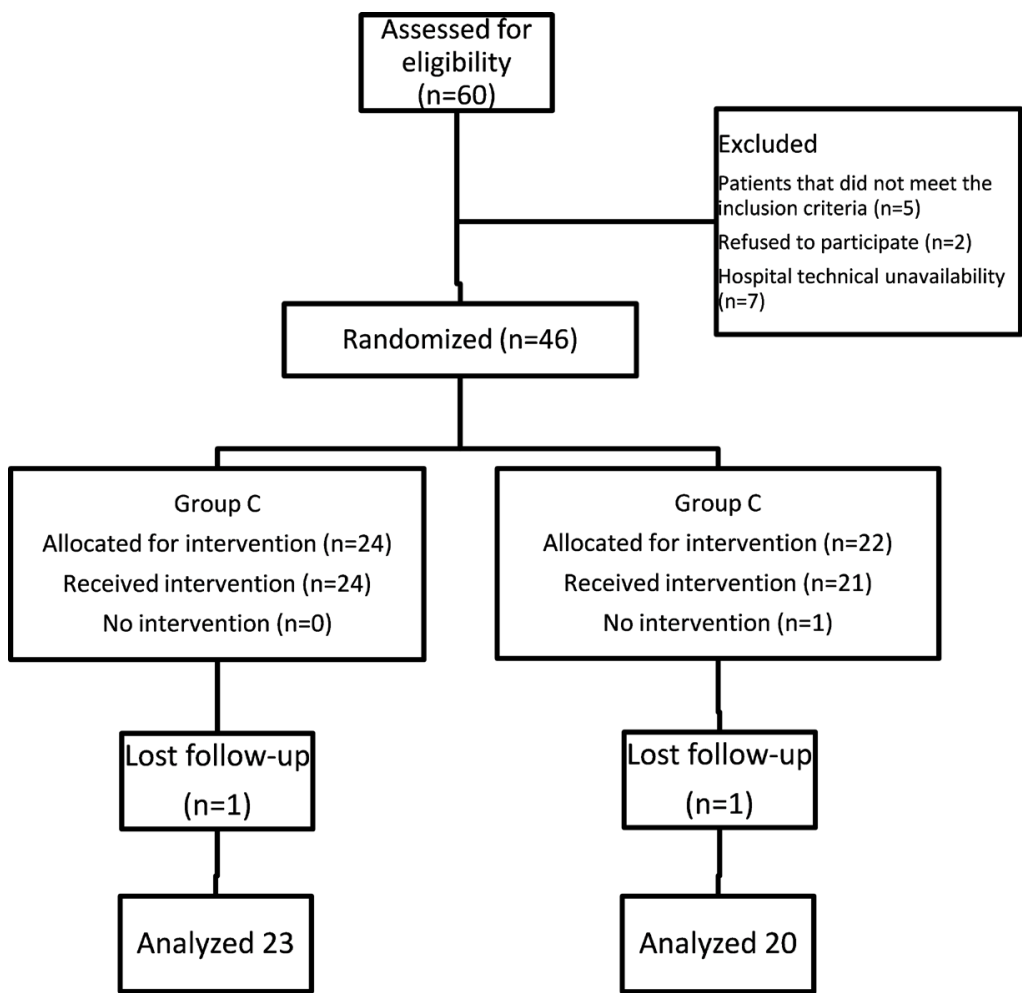

Figure 1. Flowchart of the participants. Group C (clonidine 75 to $150 \mu \mathrm{g}$ ), Group M (metoprolol 5 to $10 \mathrm{mg}$ ).

Table 1. Demographic data and clinical characteristics of patients.

\begin{tabular}{|c|c|c|c|c|}
\hline Variable & Grup C $(n=23)$ & Grup $M(n=20)$ & Total $(n=43)$ & $p$-value \\
\hline Age (years) & $49 \pm 12$ & $52 \pm 10$ & $50 \pm 11$ & 0.48 \\
\hline \multicolumn{5}{|l|}{ Sex } \\
\hline M & 10 & 11 & $21(49)$ & \multirow{2}{*}{0.65} \\
\hline $\mathrm{F}$ & 13 & 9 & $22(51)$ & \\
\hline Weight (Kg) & $72 \pm 14$ & $72 \pm 13$ & $72 \pm 13$ & 0.93 \\
\hline Height (m) & $1.61 \pm 0.1$ & $1.60 \pm 0.1$ & $1.60 \pm 0.1$ & 0.81 \\
\hline \multicolumn{5}{|l|}{ ASA } \\
\hline I & 6 & 3 & $9(21)$ & 0.46 \\
\hline II & 17 & 17 & $34(79)$ & \\
\hline \multicolumn{5}{|l|}{ Comorbities } \\
\hline Hypertension & 10 & 9 & 19 & 0.57 \\
\hline Diabetes & 7 & 4 & 11 & 0.43 \\
\hline Other & 5 & 7 & 12 & 0.32 \\
\hline Second dose & 2 & 10 & 12 & $<0.01$ \\
\hline Surgical time (min) & $154 \pm 60$ & $148 \pm 66$ & $152 \pm 62$ & 0.75 \\
\hline
\end{tabular}

Data expressed as means \pm standard deviations, or number (relative frequency). ASA, physical status according to the classification of the American Society of Anesthesiologists (ASA). Group C-clonidine, Group $\mathrm{M}-$ metoprolol. $\mathrm{P}<0.05$, statistical difference between Groups $\mathrm{M}$ and $\mathrm{C}$. 
Table 2. Pressure in the immediate post-operative period (arrival at the PACU).

\begin{tabular}{cccc}
\hline & Grup C & Grup M & $p$-value \\
\hline Initial SBP (mmHg) & $148 \pm 11$ & $151 \pm 14$ & 0.45 \\
Initial DBP (mmHg) & $91 \pm 11$ & $90 \pm 10$ & 0.66 \\
Initial HR (bpm) & $81 \pm 14$ & $83 \pm 14$ & 0.67 \\
PACU SBP (mmHg) & $127 \pm 18$ & $137 \pm 14$ & 0.03 \\
PACU DBP (mmHg) & $73 \pm 15$ & $82 \pm 7$ & $<0.01$ \\
PACU HR (bpm) & $73 \pm 16$ & $74 \pm 9$ & 0.43 \\
SBP 4h (mmHg) & $125 \pm 15$ & $128 \pm 15$ & 0.32 \\
DBP 4h (mmHg) & $79 \pm 11$ & $84 \pm 11$ & 0.88 \\
HR 4h (bpm) & $77 \pm 12$ & $76 \pm 10$ & 0.42 \\
\hline
\end{tabular}

Data expressed as means \pm standard deviations. Blood pressure in mmHg. Heart rate in beats per minute (bpm). Group $\mathrm{C}-$ clonidine, Group $\mathrm{M}-$ metoprolol. $\mathrm{P}<0.05$, statistical difference between Groups $\mathrm{M}$ and C.

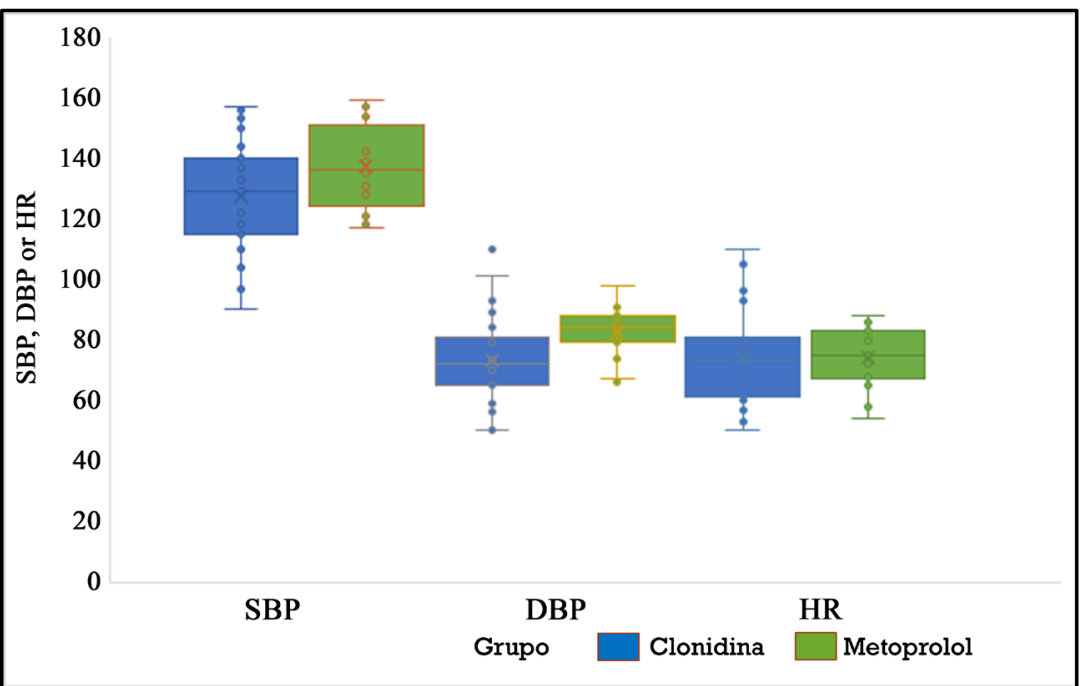

SBP, systolic blood pressure $(\mathrm{mmHg})$; DBP, diastolic blood pressure $(\mathrm{mmHg})$; HR, heart rate (bpm, beats per minute).

Figure 2. Box diagrams of blood pressure and heart rate, upon arrival at PACU.

CI: 1 - 18; $\mathrm{p}=0.03)$. Similarly, the mean DBP in group C $(73 \pm 15 \mathrm{mmHg})$ was lower than in group $\mathrm{M}(82 \pm 7 \mathrm{mmHg})$, (difference in means: $9 \mathrm{mmHg}$; $95 \%$ CI: 3 - 16; $\mathrm{p}<0.01)$. No difference was observed in the HR upon arrival at the PACU as well as in the SBP, DBP or HR four hours post-surgery (Table 2 and Figure 2).

Table 2 shows that most patients came in presenting with SBP less than 140 and DBP less than 90, with no statistical difference between groups. Among the complications observed, two patients in the clonidine group had intra-operative bronchospasm and one patient in the metoprolol group had excessive postoperative bleeding requiring urgent reoperation. No post-operative hypotension or bradycardia was observed. Vasopressors or atropine were also not used in any patients. 


\section{Discussion}

Adequate blood pressure control after OLHN surgeries is essential to prevent hemorrhagic complications. In this study, we found that metoprolol was less effective than clonidine, with higher mean values of systolic and diastolic blood pressure.

Metoprolol also presented, in this study, a disadvantage regarding the need for a second dose to control blood pressure in the pre-operative period. This happened in $50 \%$ of the patients in the metoprolol group, compared to only $8.6 \%$ of the patients in the clonidine group. This new administration implies greater dose difficulties, higher costs and less safety, since $10 \mathrm{mg}$ of metoprolol represents $75 \%$ of the maximum recommended dose (MRD), while $150 \mu \mathrm{g}$ of clonidine corresponds to only $33 \%$ of the MRD [12] [13].

Several studies have evaluated the use of both clonidine and metoprolol for blood pressure control and HR in the intra-operative period. Few studies aimed to evaluate these hemodynamic parameters in the post-operative period [14] [15]. This scarce absence of studies can be justified through consideration of the standard of care provided in developed countries, or in major medical centers. If in an ideal scenario, we are able to monitor the patient well in the post-operative period, medicating them, when necessary, quickly and effectively, anesthesiologists would be more unconcerned with possible arterial hypertension minutes or hours after the end of the surgery. Unfortunately, this is not a reality in many places, which highlights the importance of the present study, with new information to assist in the decision-making of anesthesiologists.

In a retrospective study of 641 patients who underwent nasal endoscopic surgery, a history of hypertension was a factor associated with post-operative bleeding, reaching an incidence of 8.4\% [4]. However, we did not find any studies in the literature evaluating the ideal post-operative $\mathrm{BP}$ and $\mathrm{HR}$ value in order to avoid hemorrhagic complications.

In regard to aesthetic facial surgeries, a retrospective study involving 1089 patients, observed an incidence of hematoma of $0.9 \%$ of patients. Among these, $80 \%$ had documented hypertension (systolic blood pressure $>140 \mathrm{mmHg}$ ) in the post-anesthetic care unit [5].

In thyroidectomy surgeries, in a study involving 205 patients, it was observed that 9 patients (4.4\%) had post-operative hemorrhage. Among these, $6(3 \%)$ required reoperation to empty the hematoma. Hypertension was an independent risk factor for bleeding, specifically SBP greater than $180 \mathrm{mmHg}$ [16].

In our study, in both groups, most patients had SBP less than 140 and DBP less than 90 upon arrival at the PACU. Only one patient in the M group had excessive bleeding in the post-operative period, requiring reoperation. His blood pressure at arrival was $142 \times 85 \mathrm{mmHg}$.

There were no cases of bradycardia or hypotension in the post-operative period. In a systematic review that analyzed the effects of peri-operative use of beta-blockers in non-cardiac surgery, the incidences of hypotension $($ SBP $<90$ 
$\mathrm{mmHg})$ and bradycardia $(\mathrm{HR}<60 \mathrm{bpm})$ were higher in patients who used this class of drugs. We did not observe these effects here, possibly due to the small sample size of our study, possibly dealing with the occurrence of type 2 error [17].

There were no differences observed in the 4 hours post-surgical blood pressure values between groups. On the other hand, there was no rebound effect, with blood pressure remaining lower than initial BP (upon arrival at PACU).

Higher blood pressure levels in the metoprolol group may be related to the intra-operative use of adrenaline. An association was observed between the preoperative use of beta-blockers and infiltration of the nasal mucosa with adrenaline in nasal endoscopic surgeries. A retrospective study involving 2051 patients, observed an odds ratio of 3.33 for exaggerated hypertensive events, within 1 hour after adrenaline administration [18]. In the present study, the use of adrenaline during the intra-operative period was not analyzed.

As limitations of the study, we highlight that it is difficult to establish equivalent doses among antihypertensive drugs, as these medications vary in effectiveness according to ethnicity, age, weight, use of other antihypertensive medications, comorbidities, among others. As an attempt to reduce this problem, we established titration of drugs, with the administration of sequential doses, instead of a single dose. In addition, OLHN surgeries encompass a wide variety of procedures, which have different potentials for post-operative pain, a factor directly related to the increase in blood pressure at the PACU. Therefore, different levels of pain in the post-operative period could contribute to a heterogeneity that may have influenced the results. In addition, we did not follow-up the patients after the 4 hour post-surgical period, therefore we do not know if the BP remained the same or if there was any alteration.

Further work is needed to establish which blood pressure levels are needed in order to reduce hemorrhagic complications in OLHN surgeries. With this information, it will be possible to better estimate the dose of antihypertensive drugs, such as clonidine and metoprolol, necessary to control the blood pressure of these patients in the post-operative period.

We concluded that metoprolol was less effective than clonidine in reducing systolic and diastolic blood pressures in the immediate post-operative period of OLHN surgeries when administered intravenously in the immediate pre-operative period.

\section{Conflicts of Interest}

The authors declare no conflicts of interest regarding the publication of this paper.

\section{References}

[1] EL-Shmaa, N.S., Ezz, H.A.A. and Younes, A. (2017) Labetalol, Nitroglycerin, Controlled Hypotension, Sinus Endoscopic Surgery. Journal of Clinical Anesthesia, 3, 154-158. http://dx.doi.org/10.1016/j.jclinane.2017.03.003 
[2] Koşucu, M., Ömür, Ş., Beşir, A., Uraloğlu, M., Topbaş, M. and Livaoğlu, M. (2014) Effects of Perioperative Remifentanil with Controlled Hypotension on Intraoperative Bleeding and Postoperative Edema and Ecchymosis in Open Rhinoplasty. Journal of Craniofacial Surgery, 25, 471-475. https://doi.org/10.1097/SCS.0000000000000603

[3] Rabinowitz, M.R., Cognetti, D.M. and Nyquist, G.G. (2016) Blood-Sparing Techniques in Head and Neck Surgery. Otolaryngologic Clinics of North America, 49, 549-562. http://dx.doi.org/10.1016/j.otc.2016.02.008

[4] Zeng, R., Ai, J., Xu, Z., Li, W., Ma, Y. and Tan, G. (2014) Univariate and Multivariate Analyses for Postoperative Bleeding after Nasal Endoscopic Surgery. Acta OtoLaryngologica, 134, 520-524. https://doi.org/10.3109/00016489.2013.879739

[5] Ramanadham, S.R., Mapula, S., Costa, C., Narasimhan, K., Coleman, J.E. and Rohrich, R.J. (2015) Evolution of Hypertension Management in Face Lifting in 1089 Patients. Plastic and Reconstructive Surgery, 135, 1037-1043. https://doi.org/10.1097/PRS.0000000000001131

[6] Morton, R.P. and Vandal, A.C. (2015) Postoperative Systolic Blood Pressure as a Risk Factor for Haematoma Following Thyroid Surgery. Clinical Otolaryngology, 40, 462-467. http://doi.wiley.com/10.1111/coa.12407

[7] Sadek, A.A., Mostafa, M. and Abdel-Monem, T. (2019) Metoprolol Significantly Improves Visual Clarity and Hemodynamic Parameters during Functional Endoscopic Sinus Surgery. Biomedicine Hub, 4, 1-8. https://doi.org/10.1159/000497045

[8] Jiwanmall, M., Joselyn, A.S.and Kandasamy, S. (2017) Intravenous Clonidine as a Part of Balanced Anaesthesia for Controlled Hypotension in Functional Endoscopic Sinus Surgery: A Randomised Controled Trial Address. Indian Journal of Anaesthesia, 61, 418-423. https://doi.org/10.4103/ija.IJA_58_17

[9] Puthenveettil, N., Rajan, S., Kumar, L. and Nair, S. (2013) A Comparison of Effects of Oral Premedication with Clonidine and Metoprolol on Intraoperative Hemodynamics and Surgical Conditions during Functional Endoscopic Sinus Surgery. Anesthesia Essays and Researches, 7, 371. https://doi.org/10.4103/0259-1162.123244

[10] Nguyen, V., Tiemann, D., Park, E. and Salehi, A. (2017) Alpha-2 Agonists. Anesthesiology Clinics, 35, 233-245. https://doi.org/10.1016/j.anclin.2017.01.009

[11] Devereaux, P.J., Yang, H., Yusuf, S., Guyatt, G., Leslie, K., Villar, J.C., et al. (2008) Effects of Extended-Release Metoprolol Succinate in Patients Undergoing Non-Cardiac Surgery (Poise Trial): A Randomised Controlled Trial. The Lancet, 371, 1839-1847.

[12] Whelton, P.K., Carey, R.M., Aronow, W.S., Casey, D.E., Collins, K.J., Himmelfarb, C.D., et al. (2018) 2017 ACC/AHA/AAPA/ABC/ACPM/AGS/APhA/ASH/ASPC/ NMA/PCNA Guideline for the Prevention, Detection, Evaluation, and Management of High Blood Pressure in Adults a Report of the American College of Cardiology/American Heart Association Task Force on Clinical Practice Guidelines. Hypertension, 71, 13-115. https://doi.org/10.1161/HYP.0000000000000076

[13] January, C.T., Wann, L.S., Alpert, J.S., Calkins, H., Cigarroa, J.E., Cleveland, J.C., et al. (2014) 2014 AHA/ACC/HRS Guideline for the Management of Patients with Atrial Fibrillation: Executive Summary. Journal of the American College of Cardiology, 64, 2246-2280. http://www.onlinejacc.org/content/64/21/2246.abstract https://doi.org/10.1016/j.jacc.2014.03.021

[14] Cardesin, A., Pontes, C., Rosell, R., Escamilla, Y., Marco, J., Escobar, M.J., et al. (2015) A Randomised Double Blind Clinical Trial to Compare Surgical Field Bleeding during Endoscopic Sinus Surgery with Clonidine-Based or Remifentanil-Based Hypotensive Anaesthesia. Rhinology, 53, 107-115. 
https://doi.org/10.4193/Rhin14.185

[15] Tugrul, S., Dogan, R., Senturk, E., Kocak, I., Sezen, S., Bakan, M., et al. (2016) Effect of the Premedication with Oral Clonidine on Surgical Comfort in Patients Undergoing Fess Due to Advanced Nasal Polyposis: A Randomized Double Blind Clinical Trial. American Journal of Otolaryngology, 37, 538-543.

https://doi.org/10.1016/j.amjoto.2016.08.008

[16] Perera, M., Anabell, L., Page, D., Harding, T., Gnaneswaran, N. and Chan, S. (2016) Risk Factors for Post-Thyroidectomy Haematoma. The Journal of Laryngology \& Otology, 130, S20-S25. https://doi.org/10.1017/S0022215115003199

[17] Blessberger, H., Lewis, S.R., Pritchard, M.W., Fawcett, L.J., Domanovits, H., Schlager, O., et al. (2019) Perioperative Beta-Blockers for Preventing Surgery-Related Mortality and Morbidity in Adults Undergoing Non-Cardiac Surgery. Cochrane Library: Cochrane Reviews, 9, Article ID: CD013438.

https://doi.org/10.1002/14651858.CD013438

[18] Schechtman, S.A., Wertz, A.P., Shanks, A., Thompson, A., Tremper, K., Pynnonen, M.A., et al. (2017) Preoperative $\beta$-Blockade and Hypertension in the First Hour of Functional Endoscopic Sinus Surgery. Laryngoscope, 127, 1496-1505.

https://doi.org/10.1002/lary.26492 